\title{
VESSEL MAIN PROPULSION ENGINE PERFORMANCE EVALUATION
}

\author{
Tadeusz Borkowski, Przemysław Kowalak, Jarosław Myśków
}

\author{
Maritime University of Szczecin \\ Engineering Department \\ Waly Chrobrego Street 1-2, 70-500 Szczecin, Poland \\ tel.: +48914809400, fax: 48914809575 \\ e-mail:t.borkowski@am.szczecin.pl,p.kowalak@am.szczecin.pl,j.myskow@am.szczecin.pl
}

\begin{abstract}
In general, the performance of a ship in service is different from that obtained on shipyard sea trial. Apart from any differences due to loading conditions, and for which due correction should be made, these differences arise principally from the weather, fouling and surface deterioration of the hull and propeller. The influence of the weather, both in terms of wind and sea conditions, is an extremely important factor in ship performance analysis. Consequently, the weather effects needs to be taken into account if a realistic evaluation is to be made. The primary role of the ship service analysis is a standard of performance data, under varying operational and environmental conditions. The resulting information, derived from this data, becomes the basis for operational and chartering decision. In addition, the part for the data records is to enable the analysis of trends of either the hull or machinery, from which the identification of potential failure scenarios and maintenance decisions can be derived. The traditional method of data collection is the deck and engine room log records, and this is the most commonly used method today. In terms of data processing and capabilities, this method of data collection is far from ultimate, since involves significant data distortion risk. Instrumentation errors are always a potential source of concern in performance analysis methods. Such errors are generally in the form of instrument drift or gross distortion of the reading. However, these can generally be detected by the use of trend analysis techniques. The procedure for the evaluation of the ship's service performance, that relies on proven methods of main propulsion engine service data analysis used and applied for container vessel - small feeder. The vessel is equipped with indirect main propulsion, driven by means of modern medium speed engine. The different approach demonstrated to achieve the reliable and accurate main engine performance. The difference in developed engine power has been found, that corresponds well to registered sea trial results and engine retrofitting reports done, in order to limit the effective power.
\end{abstract}

Keywords: ship main propulsion, marine diesel engine, energy efficiency design index,

\section{Introduction}

In general, the performance of a ship in service is different from that obtained during the shipyard sea trial. Apart from any differences due to loading conditions, and for which due correction should be made, these differences arise principally from the weather condition, fouling and surface deterioration of the hull and propeller [1]. The influence of the weather, both in terms of wind and sea conditions, is an extremely important factor in ship performance analysis. Consequently, the weather effects needs to be taken into account if a realistic evaluation is to be made. The primary role of the ship service analysis is a standard of performance data, under varying operational and environmental conditions. The resulting information, derived from this data, becomes the basis for operational and chartering decision. In addition, the part for the data records is to enable the analysis of trends of either the hull or machinery, from which the identification of potential failure scenarios and maintenance decisions can be derived [2]. The traditional method of data collection is the deck and engine room log records, and this is the most commonly used technique today. In terms of data processing and capabilities, this method of data collection is far from ultimate, since involves significant data distortion risk. Instrumentation errors are always a potential source of concern in performance analysis methods. Such errors are generally in the form of instrument drift or gross distortion of the reading. However, these can 
generally be detected by the use of trend analysis techniques [3, 4]. The procedure for the evaluation of the ship's service performance, that relies on proven methods and contains service data analysis of main propulsion engine, used and applied for container vessel - small feeder, that basic details are shown in Tab. 1. The vessel is equipped with indirect main propulsion, driven by means of modern medium speed engine. The different approach demonstrated to achieve the reliable and accurate main engine performance. The main engine performance survey was carried out as a part research project and appropriate measurements conducted, during the ship's steaming at the Baltic sea. Throughout the sea trials, vessel was in loaded condition and all relevant details are given in Tab. 2.

Tab. 1. Ship main details

\begin{tabular}{|l|l|c|}
\hline No & \multicolumn{2}{|c|}{ Description } \\
\hline 1 & Class & Open top container vessel \\
\hline 2 & Length over all & $134.44 \mathrm{~m}$ \\
\hline 3 & Breadth moulded & $22.50 \mathrm{~m}$ \\
\hline 4 & Draught - design, loaded & $8.50 \mathrm{~m} / 8.71 \mathrm{~m}$ \\
\hline 5 & Deadweight & $11000 \mathrm{TDW}$ \\
\hline 6 & Container intake & $872 \mathrm{TEU}$ \\
\hline 7 & Main engine & MAK 8M43 \\
\hline
\end{tabular}

Tab. 2. Nautical and ambient condition during the sea trials

\begin{tabular}{|l|l|c|}
\hline No & \multicolumn{2}{|c|}{ Description } \\
\hline 1 & Draught (forward/afterward) & $8.2 \mathrm{~m} / 8.6 \mathrm{~m}$ \\
\hline 2 & Cargo on board & $6790.9 \mathrm{mt}$ \\
\hline 3 & Sea state - wind force and direction & $3-4^{\circ} \mathrm{B}, \mathrm{SW}$ \\
\hline 4 & Barometric pressure & $1015 \mathrm{hPa}$ \\
\hline 5 & Ambient air temperature & $16^{\circ} \mathrm{C}$ \\
\hline 6 & Shaft generator load & $270 \mathrm{~kW}$ \\
\hline
\end{tabular}

\section{The main propulsion performance evaluation - scope and procedure}

The ship speed and propulsion power analysis recommended by ITTC [5], follows a methodology ISO [6] and was utilized in the recent work. Preferably, the wind resistance coefficients of the ship should be obtained from model tests. In most cases, model tests are not available and environmental influences on the performance of sea trials, speed runs should only be performed against and with the waves. The correction methods existing so far account for the influences of waves only for these two conditions; in the case when waves do not come from the bow or the stern, the correction methods are not sufficiently reliable and the effects of steering and drift on the ship's performance might be underestimated. Hence, no corrections were utilized in analysis. Main engine performance estimation was performed under ship's sea service condition, in good weather and under loaded condition. The main engine performance, speed and torque were measured to obtain the brake effective power. The engine power estimation was attained through three alternative methods and is shown in Fig. 1.

1. engine rotational speed and torque measurement - on engine flywheel,

2. rotational speed and torque measurement - main propulsion shaft

3. engine mean indicated pressure measurement - each engine cylinder.

The electronic MIP (Mean Indicated Pressure) calculator with TDC sensor enables cylinder direct pressure measurements. With the TDC (Top Dead Centre) sensor connected, the course of pressure with relation to the crankshaft angle is measured thus enabling calculation of indicated power and extensive combustion analysis. Powerful analysis software allows the computation of 
power for each engine cylinder. For the purpose of main engine performance, evaluation the ship engine standard monitoring system was utilized. Shaft torque measurements are generally conducted with a full-bridge strain gauge rosette excited with a battery box and amplified with a purpose built amplifier-decoder and transmitted to stationary receiver through antenna as shown in Fig. 2. Calibration is performed by placing a shunt resistor into one of the arms of strain gauge bridge, which simulates corresponding strain. The uncertainty of this measurement system consists of elemental error sources based on: a strain gauge, calibration of measurement system, installation on a ship and torque calculation. Shaft speed measurements are made with magnetic pulse sensor and a frequency counter.

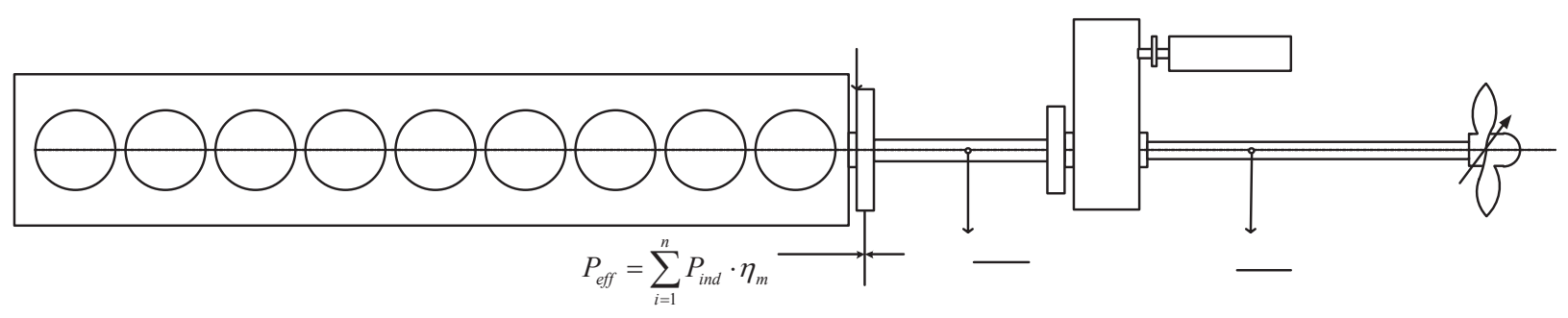

Fig. 1. Main engine and propulsion effective power evaluation

The number of pulses are counted for a predetermined time and divided into number pulses per revolution to find shaft rate of revolution. Bias error in pulse count is 1 pulse, there is no uncertainty in revolution estimation. As time window gets larger, the bias error associated with shaft rate of revolution drops. However, then the transient changes in the shaft power are not acquired. For the current work, time window is taken as $1 \mathrm{~s}$, as power is calculated once every second. The propulsion shaft torque measurement telemetry system (presented in Fig. 2, with specification in Tab. 3) offers wireless transmission of strain gauge signals from rotating shafts.

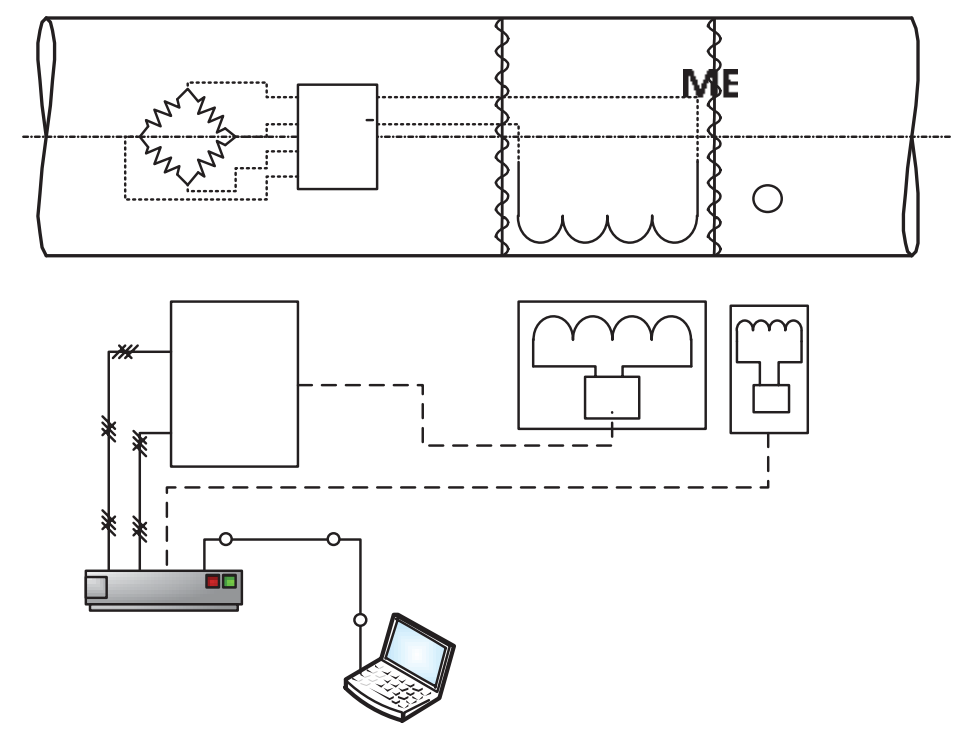

Fig. 2. The main engine and propulsion effective power measurement setup

The recorded torque data were recalculated into power with aid of a rotational speed signal collected from the pickup sensors, which are built in torque meters acquisition system. The main engine performance has been recorded for further analysis that includes:

- continuous engine output shaft torque - speed profile,

- engine cylinder pressure data,

- engine fuel oil consumption, 
- engine operational condition parameters.

Tab. 3. Experimental instrumentation details

\begin{tabular}{|l|c|}
\hline \multicolumn{1}{|c|}{ Specification } & Instrument \\
\hline Engine torque and rotational speed & $\begin{array}{c}\text { Strain gauge type, wireless torque meter, Krauss, type: } \\
\text { TEL1-PCM-STG }\end{array}$ \\
\hline Propulsion shaft torque and rotational speed & $\begin{array}{c}\text { Strain gauge type, wireless torque meter, Philips, type: } \\
\text { PR9910 }\end{array}$ \\
\hline Indicated cylinder pressure and power & Digital pressure indicator, Leutert, type: DPI-2 \\
\hline
\end{tabular}

\section{Results and discussion}

The experimental data sets were collected for several different engine load levels, ranging from approximately $25 \%$ of nominal load and up to the maximum acceptable limit for engine operation. Main propulsion load profile was created as an effect of ship's accelerating from dead slow to full ahead that is presented in Fig. 3. The main propulsion overload can be achieved only by means of controllable pitch propeller setting. The maximum engine effective power was reached when limits position of the injection pumps fuel racks and controllable pitch propeller overload system activated.

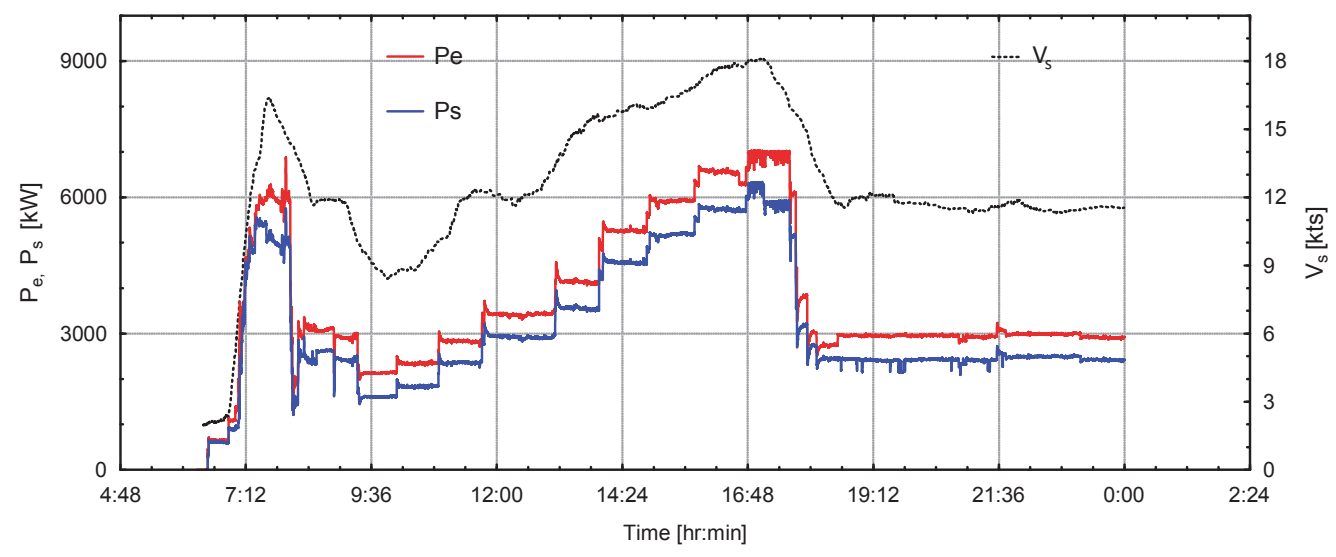

Fig. 3. Main propulsion power demanded by ship's acceleration

The recorded data were processed, and extracted results are presented in form of the graphs. The Fig. 4, is meant to compare the actual engine performance with the test bed results, listed in manufacturer engine shop trial report. The engine specific fuel oil consumption, cylinder maximum combustion pressure and turbocharger rotational speed was utilized to validate the experimental torque - speed data set and measurement methodology. Possible discrepancies between test bed and sea trials results may indicate improper acquisition installation or other signal disturbances.
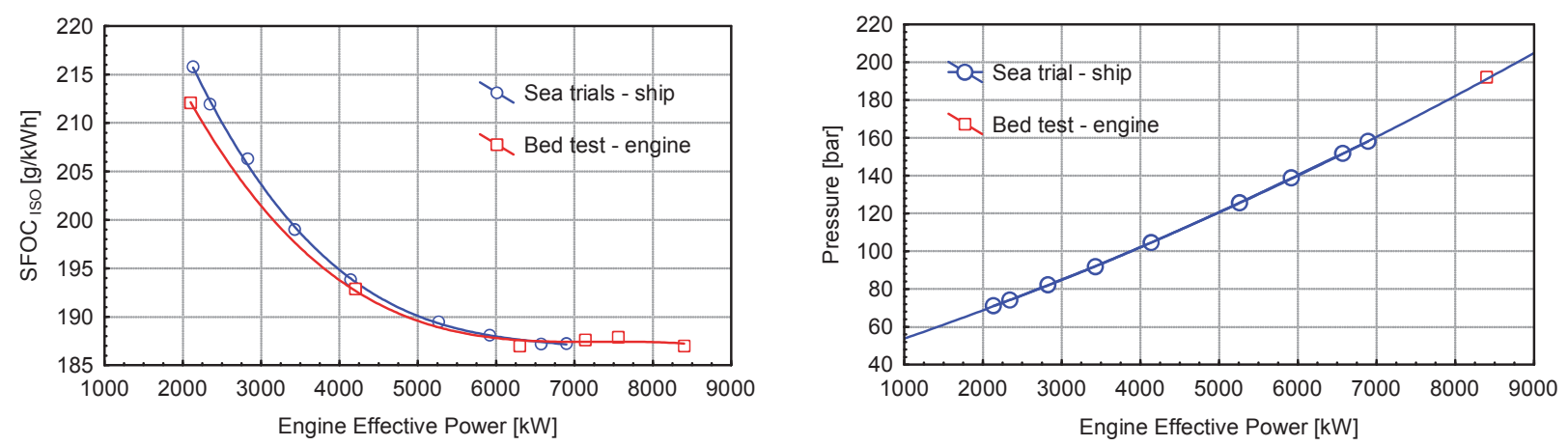

Fig. 4. Engine effective power versus SFOC (left) and maximum combustion pressure (right) 
Based on the engine performance comparison presented in Fig. 4, the measurement methodology and experimental installation were appraised as free from significant methodology and experimental installation inaccuracies. It can be noticed that the engine maintains the test bed performance and operational factors, even after 18000 running hours and over 5 years of exploitation, that confirms engine excellent condition. The diagram in Fig. 5 presents the engine effective power comparison derived from the four different sources, caused by controllable pitch propeller setting increase and relevant ship acceleration.

The engine calculated mechanical efficiency varies from $78 \%$ at low load (approximately 25\% of rated power) to $91 \%$ at high load (approximately $78 \%$ of rated power). Such set of values reflects expected result for modern large bore marine four-stroke and medium speed diesel engine. It can be noticed that the engine's control system - CAT Dicare, presents values reflecting indicated power rather than the engine's effective power as it can be expected.

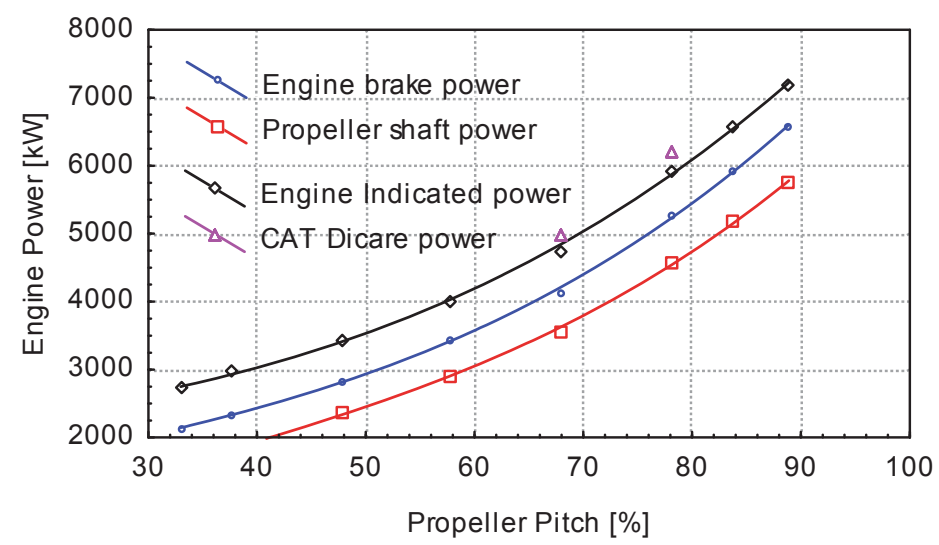

Fig. 5. The engine and shaft power comparison

The diagram on Fig. 6 shows three engine's fuel rack indicators comparison with different alignment, which is adequate to engine developed power. Adjustment of engine control local indicator and fuel injection pumps are almost parallel to the readings taken during test bed trial. However, indication given by CAT Dicare system has different span (inclination). The most important aspect is related to the maximum available engine effective power, which was reached with local indicator position of $52 \mathrm{~mm}$. This position reflects engine nominal load and is confirmed by the results observed during test bed operation

However, the maximum developed engine effective power, during the sea trial (reflecting to the local rack position of $52 \mathrm{~mm}$ ) was only $6950 \mathrm{~kW}$, despite the test bed result where the engine developed rated power was $8400 \mathrm{~kW}$, with the same fuel rack setting $-52 \mathrm{~mm}$.

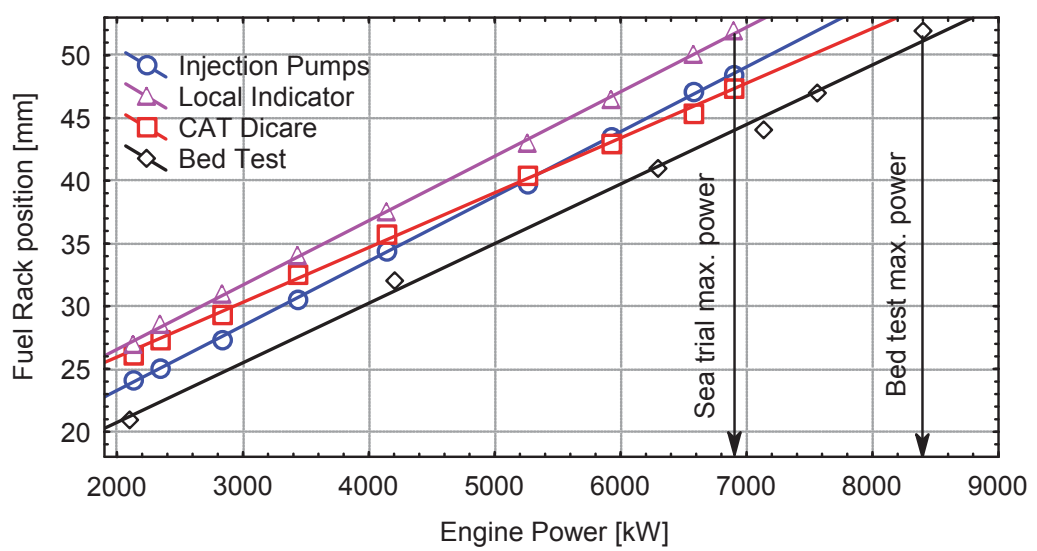

Fig. 6. The engine's fuel rack indicators comparison in useful engine load range 
The fuel oil grade has to be taken into account if relation engine effective power and fuel rack settings is analysed. Due to the obvious differences of the fuel grades used during sea trials and test bed operation which presents Tab. 4, expected fuel rack position might slightly vary from the one reached during the test bed trial. This distinction could be calculated, following the fuel oil property.

The fuel injection pump represents volumetric positive displacement type and the volumetric calorific value of the fuel rate should be taken into account based on the expression:

$$
W_{v}=W_{s} \cdot \rho_{\text {ivijection }}\left[\frac{\mathrm{kj}}{\mathrm{dm}}=\frac{\mathrm{kJ}}{\mathrm{kg}} \cdot \frac{\mathrm{kg}}{\mathrm{dm}}\right] \text {, }
$$

where:

$W_{v}$ - fuel oil volumetric calorific value,

$W_{s}$ - fuel oil specific calorific value,

$P_{\text {injection }}$ - delivered fuel oil density.

Tab. 4. Fuel oil property comparison

\begin{tabular}{|l|c|c|}
\hline Determination & Test bed & Sea trial \\
\hline Fuel oil density @ $15^{\circ} \mathrm{C}$ & $830 \mathrm{~kg} / \mathrm{m}^{3}$ & $991 \mathrm{~kg} / \mathrm{m}^{3}$ \\
\hline Fuel oil lower heating calorific value & $42232 \mathrm{~kJ} / \mathrm{kg}$ & $40380 \mathrm{~kJ} / \mathrm{kg}$ \\
\hline Delivered fuel oil temperature & $50^{\circ} \mathrm{C}(\mathrm{estimated})$ & $132^{\circ} \mathrm{C}$ \\
\hline Delivered fuel oil density & $807.3 \mathrm{~kg} / \mathrm{m}^{3}$ & $917.9 \mathrm{~kg} / \mathrm{m}^{3}$ \\
\hline
\end{tabular}

The corrected volumetric calorific rates for the fuel oil in service - sea trials and test bed are presented in Tab. 5. Subsequently, based on the LHV data for both fuel grades, the volumetric correction factor can be derived. This factor can be utilized for further estimation of the expected fuel rack position for engine heavy fuel oil operation.

$$
C_{v}=\frac{W_{v M G O}}{\mathrm{~W}_{\mathrm{vHFO}}},
$$

where:

$C_{v} \quad-$ volumetric correction factor,

$W_{v M G O}$ - volumetric calorific value for MGO,

$W_{v H F O}$ - volumetric calorific value for HFO.

Tab. 5. Fuel oil volumetric calorific values and volumetric correction factor

\begin{tabular}{|c|c|c|c|}
\hline Temperature at injection & Test bed $W_{v}$ & Sea trials $W_{v}$ & $C_{v}$ \\
\hline $20^{\circ} \mathrm{C}$ & $34917 \mathrm{~kJ} / \mathrm{dm}^{3}$ & - & 0.9421 \\
\hline $30^{\circ} \mathrm{C}$ & $34643 \mathrm{~kJ} / \mathrm{dm}^{3}$ & - & 0.9347 \\
\hline $40^{\circ} \mathrm{C}$ & $34368 \mathrm{~kJ} / \mathrm{dm}^{3}$ & - & 0.9273 \\
\hline $50^{\circ} \mathrm{C}$ & $34094 \mathrm{~kJ} / \mathrm{dm}^{3}$ & - & 0.9199 \\
\hline $60^{\circ} \mathrm{C}$ & $33819 \mathrm{~kJ} / \mathrm{dm}^{3}$ & - & 0.9125 \\
\hline $132^{\circ} \mathrm{C}$ & - & $37064 \mathrm{~kJ} / \mathrm{dm}^{3}$ & 1 \\
\hline
\end{tabular}


Finally, the estimated engine fuel rack settings set against realistic sea trials and test bed operation data set is presented in Fig. 7. The highest line (local indicator), injection pumps, and Cat Dicare sets denotes engine sea trial results and substantial discrepancy needs to be emphasized. Thus, it can be pointed that the sea trials engine rack positions are far from the expectation.

The estimated injection pump rack position tendency, caused by fuel oil grade was confirmed by the engine maker: "When using heavier fuel grades independent on the density of the fuel, the rated power will be reached at a fuel rack position that is accordingly lower", (engine manual).

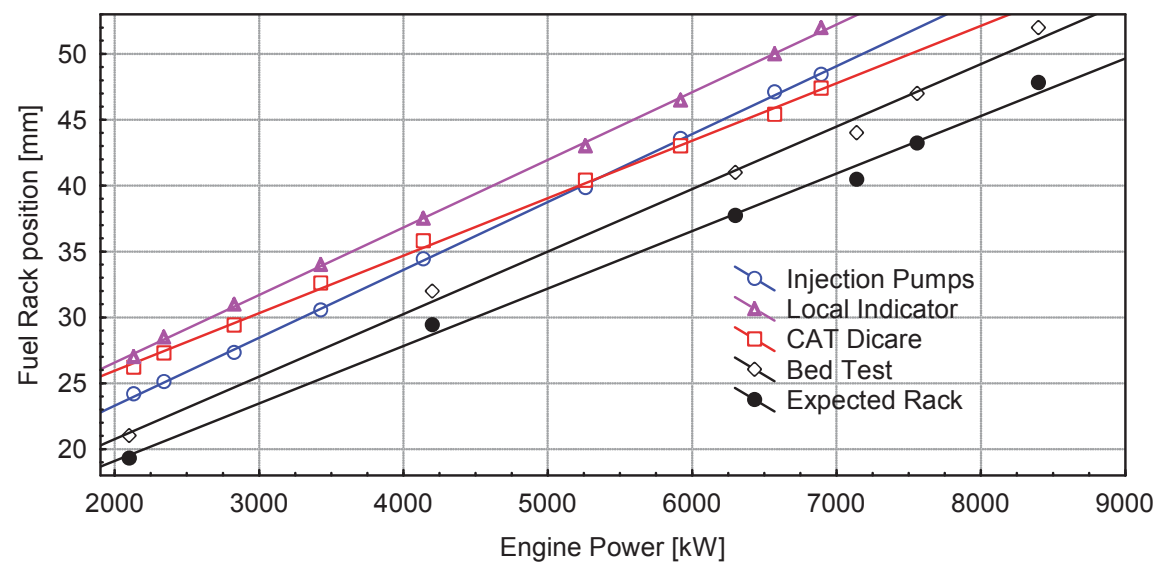

Fig. 7. The engine's fuel rack indicators comparison

\section{Conclusions}

The comparative process of realistic main engine performance related to the effective power (independently from the fuel rack position) with the test bed shop trial report results provides clear and consistent view of engine actual condition. Basic set of engine performance factors expressed by effective power and fuel consumption shows reasonable agreement with factory performance report. It can be noticed that all recorded engine operational parameters are very close to the new state. Especially, cylinder maximum combustion pressure follows the values taken during the engine shop trial test. It indicates that the cylinder combustion process is not altered significantly. However, due to fuel rack position shift engine is not capable to develop the service and rated power. Currently, engine fuel rack settings at $52 \mathrm{~mm}$ caused $6950 \mathrm{~kW}$ of engine developed effective power, whilst estimation is $8400 \mathrm{~kW}$, with fuel rack position of $48 \mathrm{~mm}$. Such enginereduced output may lead to difficulties in achieving the ship's service or contract speed. During sea trials, the maximum available propeller's pitch was $93 \%$ with shaft generator disconnected. When steaming with higher amount of reefer containers the maximum available pitch might be much lower and therefore the vessel's required speed cannot be maintained.

The engine effective power evaluated by the engine's control system - CAT Dicare arrangement is far from correctness. Almost parallel course of the sea trial engine rack position with the test bed results specifies fuel injection pumps assembly incorrectness. Hypothetically, similar situation may appear when in all fuel injection pumps the control sleeve is misaligned with control rod by the same value. Another hypothetical reason might be installation of the plungers with different angle of the control edge than that installed during bed test. The above-mentioned possible reasons and solutions should be understand as possible and hypothetical only and should be consulted with the engine maker before the further action.

\section{References}

[1] Min, K., Kang, S., Study on the form factor and full-scale ship resistance prediction method, Journal of Marine Science Technology, 15:108-118, DOI 10.1007/s00773-009-0077-y, 2010. 
[2] Andresen, P., Borrod, A. Blanchot, H., Evaluation of the Service Performance of Ships, Marine Technology and SNAME News, 42,4 ProQuest Central pg. 177, 2005.

[3] Hountalas, D. T., Prediction of marine diesel engine performance under fault conditions, Applied Thermal Engineering 20, 1753-1783, www.elsevier.com/locate/apthermeng, 2000.

[4] Insel, M., Uncertainty in the analysis of speed and powering trials, Ocean Engineering 35, 1183-1193, Elsvier, 2008.

[5] International Towing Tank Conference, Recommended Procedures and Guidelines, Testing and Extrapolation Methods Loads and Responses, Stability Model Tests on Intact Stability, UK 2005.

[6] ISO 15016:2002, Ships and marine technology - Guidelines for the assessment of speed and power performance by analysis of speed trial data. 